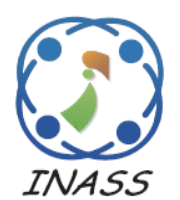

\title{
A Cognitive Agent Model of Burnout for Front-line Healthcare Professionals in Times of COVID-19 Pandemic
}

\author{
Hayder M. A. Ghanimi ${ }^{1^{*}}$ \\ Shaymah Akram Yasear ${ }^{2}$ \\ ${ }^{l}$ Department of Biomedical Engineering, College of Engineering, University of Warith Al-Anbiyaa, \\ Karbala 56001, Iraq \\ ${ }^{2}$ Department of Biomedical Engineering, Al-Mustaqbal University College, Babil, Iraq \\ * Corresponding author's Email: hayder.alghanami@uowa.edu.iq
}

\begin{abstract}
The global pandemic (Covid-2019) has severely affected all aspects of our life and even changed the way we live and work. As the pandemic outspread, healthcare professionals need urgent interventions to control the harmful consequences. Encountering such a crisis makes them more prone to negative psychological ramifications in their work environment, making them unable to provide the proper support. Burnout is the most negative feeling increased among healthcare professionals while compacting the virus. One essential move to remedy the impacts of burnout is understanding its determinants and their causal relationships. This paper addresses the design of a computational (cognitive) agent model of burnout for healthcare professionals using a temporal-causal network model. Several determinants of burnout with their causal relationships were identified from the literature and formalised to construct the proposed cognitive agent model. In addition, different simulation experiments were implemented to obtain a clear insight into the causal relationships among burnout determinants and those experiments are exhibited similar behaviours to exiting literature. Furthermore, the developed model was evaluated using two different methods: mathematical analysis to prove its implementation was achieved right and automated logical verification to check several properties as shown in the literature to confirm that the suitable model was built. The obtained cognitive agent model could be helpful to develop a covid-19-aware analytics software agent that can monitor healthcare professionals' mental health.
\end{abstract}

Keywords: COVID-19 pandemic, Agent-based model, Stress, Network oriented modelling, Computational modelling.

\section{Introduction}

The coronavirus or COVID-19 global pandemic has primarily affected our lives, and its harmful consequences are enormous. It impacted most life domains and sectors, in which the health sector was not an exception. As of 22 September 2021, world health organization (WHO) declared that 229,373,963 million people had been infected with the virus, with more than 4.7 million deaths worldwide [1]. Since the emergence of the pandemic, the impacts on healthcare professionals have been numerous, especially in countries that had not experienced similar epidemic outbreaks in recent years [2]. The imposed effects are related to increased workload and the immense and unprecedented pressure, threatening their physical, mental, and social well-being. It is a fact that covid-19 is unlike previous pandemics in terms of its impacts as it might be more challenging because of its unique features such as the high contagiousness, a relatively low level of knowledge regarding the course of infection and (long-term) consequences, and a lack of established treatments or vaccines [3]. Studies on the adverse psychological effects of covid-19 on healthcare professionals (i.e., those in direct contact with infected patients) have shown that front-line workers can have many harmful consequences on their emotional and mental well-being [4]. Dangerous psychological symptoms such as stress and anxiety are well-known examples that negatively impact the psychological well-being of front-line healthcare professionals. An initial finding from a study 
conducted in October 2020 by the British medical association found that $43.7 \%$ of participating doctors were suffering from several symptoms of stress, anxiety, and emotional distress during their medical practice amid the current pandemic [5].

Furthermore, continuous exposure to these harmful conditions could develop a state of emotional and physical exhaustion that leads to mental health problems. It affects the ability to manage their performance at work. This unwanted and exhausting state of front-line workers is called burnout [6]. Burnout is a chronic psychological condition with a loss of enthusiasm and personal accomplishment, feelings of physical and mental exhaustion, and depersonalisation [7]. While struggling against covid-19, this harmful condition of burnout for healthcare professionals has become a significant concern. Due to this concern, it has become more important to know the causes of burnout and how to prevent, avoid, and, if possible, cure them. Previous studies have been conducted to determine the prevalence of and factors related to burnout $[8,9]$. Therefore, in a bid to control or remedy the condition of burnout, a cognitive agent model of burnout based on the causal relationships among its determinants is necessary to be developed. Previous efforts have been made to create dynamic models of burnout to understand the dynamic causal interplays between its factors [9]. However, these developed models are not covering some essential components such as the psychological developed burden and resilience of healthcare professionals while compacting the covid19.

Moreover, with the emergence of covid-19, the scientific community has made a clear call for more robust computational models to understand the new conditions under crisis [10]. Therefore, this paper aims at designing a cognitive agent model of burnout based on the dynamic causal interactions among burnout's determinants to suit the domain of healthcare workers at the covid-19 crises.

To do so, causal modelling (the network oriented modelling approach) will be helpful. Note here that this modelling approach based on a temporal causal network is similar to other causal modelling approaches. However, it has several merits that make this approach our modelling choice. For example, incorporating a continuous-time dimension to model dynamics enables causal reasoning and simulation for cyclic causal graphs. A complete description of network-oriented modelling merits can be seen in [11]. Notably, the network-oriented modelling approach based on the temporal causal network has witnessed substantial progress in modelling human cognitive states and processes. This approach has been and is being used successfully to understand and get profound insights into the complexity of the human mind. Therefore, reasoning ability becomes possible to be supplied while creating intelligent applications/systems [12].

Developing dynamic models for human mental states and processes in a computational form and integrating them into intelligent applications (i.e., a virtual agent or robot) will allow such intelligent agents to perform reasoning and analysis in a more human-like manner. This capability enables agents to provide intelligent support in the same way as a human [13]. In other words, intelligent agents (i.e., software/hardware agents) are usually developed to provide personal care depending upon acquiring sensor information about human and their environment, but creating more intelligent agents can be achieved through the use of knowledge to perform depth analysis of human functioning. Suppose this knowledge of human functioning is represented in the form of a causal network in an ambient agent. In that case, the agent will be capable of providing support knowledgeably and eventually help in improving the human's well-being and performance $[14,15]$.

The reasons above are enough to justify why this modelling approach is good to be implemented to develop a cognitive agent model of burnout.

The rest of this paper is organised as follows. Section 2 describes the fundamentals psychological concepts of burnout and finds out its major determinants with their interactions. The designed cognitive model based on the identified factors from the previous section is discussed in section 3 . Furthermore, details on the network-oriented modelling approach are presented. Section 4 illustrates the simulation experiments of the designed model. Next, the developed cognitive model is mathematically evaluated in section 5. Section 6 discusses the internal validity of the model using automated logical verification. Finally, section 7 concludes the paper.

\section{Conceptual development of burnout}

Since burnout rates have been rising, much attention paid to obtain insights into its development. Burnout is a common occupational and public-health problem. Specifically, burnout is explained as the feelings of emotional and physical exhaustion and also described as the feeling of negativism or cynicism towards one's job [6]. Increment in the burnout level has serious negative consequences as it contributes to a decrease in job performance and job attendance. Furthermore, burnout has adverse 


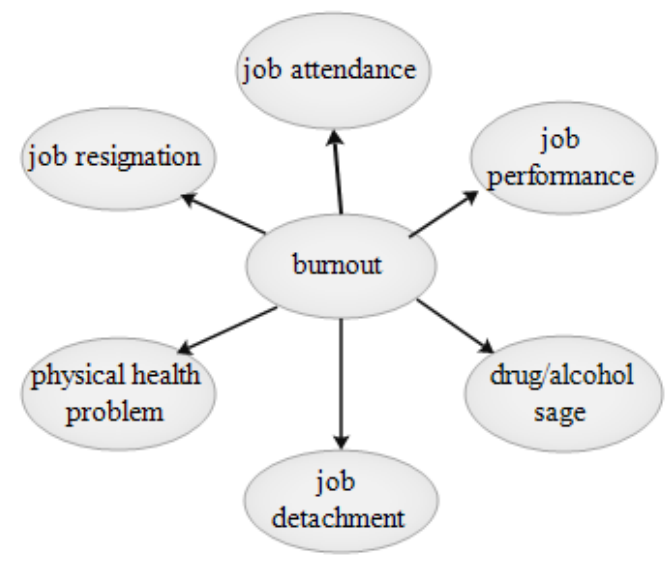

Figure. 1 Burnout consequences

impacts on people physical health, and it shows, for example, in the form of weight fluctuations. These consequent destructive states are adequately illustrated by [8] and depicted in Fig. 1.

It is well-known that the stressful event (i.e., the covid-19 pandemic) requires mental and physical efforts to handle its impact (i.e., situational demands) [16]. Moreover, it puts severe pressure on front-line workers that threaten their physical, mental, and social well-being. Healthcare professionals facing such dangerous events experience an increased physical and psychological burden, which is considered a risk of burnout [17].

Covid-19 placed a significant amount of untenable burden on front-line workers as they had no choice but to fight the virus and put their health and lives at risk to support and treat affected people. Many reasons such as lockdown, no clear information about the virus seriousness and redemption, enough places to accommodate the patients, and available protective types of equipment represent the missing resources that healthcare workers need to meet the psychological and physical burden imposed by covid-19 [3].

Another crucial psychological concept related to burnout development is resilience. It is defined as "the capacity of individuals to cope successfully with significant change, adversity or risk" [18]. It explains why some healthcare workers are less affected by burnout and other harmful consequences. It is important to mention that resilience is a critical factor that controls the increase in burnout [19]. Psychological resilience is also related to one's belief towards achieving its goal (self-efficacy) and one's personality. Both self-efficacy and positive personality (e.g., openness) were found to be positively correlated with resilience [20-21].

More importantly, some healthcare professionals who are emotionally and physically exhausted due to the impact of covid-19 tend to distant themselves and disengage from work (cynicism) [6]. This scenario can be seen when they have no motivation to manage the burden and emotional and physical exhaustion resulting from while treating the patients. Social contact or social support from friends, colleagues, and family members is essential in motivating healthcare professionals to continue providing necessary interventions to patients. However, healthcare professionals often neglect relationships with their friends and family due to heavy workloads or concerns around infecting others due to their own occupational exposure to the virus. Moreover, maintenance of social contact is increasingly challenging in the context of social distancing requirements and, anecdotally, there are reports of healthcare workers experiencing social stigma and abuse due to public fears of contracting the virus from those with the most significant exposure. To sum up, social contact/support is an issue that contributes negatively to burnout [22].

In addition, the literature has paid much attention to the importance of coping strategies in the development or reduction of burnout [23]. When front-line professionals experience stressful and burdening events that develop burnout, they rely on their coping mechanisms to handle the negative consequences. These coping strategies are categories into problem-focused or emotion-focused; adaptive or maladaptive cognitive/behavioural strategies. Many research works have revealed that front-line workers who apply problem-focused coping strategies tend to reduce the negative impact of stress, emotional exhaustion, and burnout [24]. On the contrary, those who implement emotion-focused coping strategies are experiencing a high level of burden resulting an increment in stress level and emotional exhaustion in which correlated positively to the development of burnout [25].

All the above causal concepts are necessary to design a graphical representation of the cognitive agent model of burnout, as discussed in the next section.

\section{Cognitive agent model of burnout}

This section illustrates the development process of the cognitive model of burnout, which consists of presenting the model's conceptual design (in a form of a causal graph) and the formalisation process (in a form of differential equations). Prior to that, detailed explanations related to the network-oriented modelling approach are addressed. 
Table 1. Temporal causal network specifications

\begin{tabular}{|c|c|c|}
\hline Concept & Conceptual Representation & Explanations \\
\hline $\begin{array}{l}\text { states symbols } \\
\text { and their } \\
\text { connections }\end{array}$ & $X, Y, X \rightarrow Y$ & $\begin{array}{l}\text { Describes the representation of network states/nodes with their } \\
\text { links in a graphical form. }\end{array}$ \\
\hline $\begin{array}{l}\text { Connection } \\
\text { weight }\end{array}$ & $\omega_{x, y}$ & $\begin{array}{l}\text { The connection weight between two states }(X \text { and } Y) \text {. It } \\
\text { describes the strength level of the causal impact of state } X \text { on } \\
\text { state } Y \text { through connection } X \rightarrow Y \text {. It is usually in a range } \\
\text { between zero and one }[0,1] \text {. }\end{array}$ \\
\hline $\begin{array}{l}\text { Combining } \\
\text { multiple } \\
\text { impacts on a } \\
\text { state }\end{array}$ & $\mathrm{c}_{Y}(.)$. & $\begin{array}{l}\text { To measure the level of state } Y \text {, a combination function } \mathrm{c}_{Y}(. .) \text { is } \\
\text { derived from aggregating the causal impacts of other states on } \\
\text { state } Y \text {. }\end{array}$ \\
\hline Speed Factor & $\eta_{Y}$ & $\begin{array}{l}\text { This describes how fast state } Y \text { can be influenced by the } \\
\text { combined causal impacts from the incoming connections. The } \\
\text { range of the speed is generally between } 0 \text { and } 1 \text {, representing } \\
\text { low and high, respectively. }\end{array}$ \\
\hline Concept & Numerical notation & Explanations \\
\hline $\begin{array}{l}\text { State values } \\
\text { with respect to } \\
\text { time } t\end{array}$ & $Y(t)$ & $\begin{array}{l}\text { Each node (state } Y \text { ) in the model at every time point } t \text { has a } \\
\text { value ranging between } 0 \text { and } 1 \text {. }\end{array}$ \\
\hline $\begin{array}{l}\text { Single causal } \\
\text { impact on state } \\
Y \text { via an } \\
\text { incoming link } \\
\text { from state } X \\
\end{array}$ & $\operatorname{impact}_{X, Y}(t)=\omega_{x, y} X(t)$ & $\begin{array}{l}\text { At time point } t \text {, state } X \text { with a link to state } Y \text { has an impact on } \\
\text { state } Y \text {. This single impact is represented using the connection } \\
\text { weight } \omega_{x, y} \text {. }\end{array}$ \\
\hline $\begin{array}{l}\text { Aggregation: } \\
\text { Multiple causal } \\
\text { impacts }\end{array}$ & 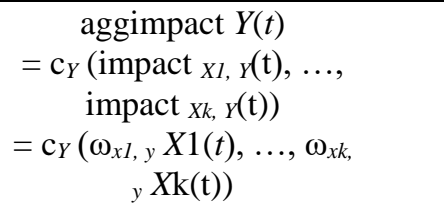 & $\begin{array}{l}\text { This represents the computation of multiple impacts on state } Y \\
\text { from all of the incoming connections from various states } X_{i} \text {. }\end{array}$ \\
\hline
\end{tabular}

\subsection{Network oriented modelling}

The network oriented modelling technique based on the temporal casual relationships is implemented to design the cognitive agent model of burnout [11], [26]. This technique is used in a form of a network whereby each node/state of the network has a specific characteristic that can change over time. To explain the working mechanism of this technique, each node $X$ is linked to another node $Y$ via a connection that carries causal impact from $X$ to $Y$. This illustrates the causal relationships applied by $X$ on $Y$. The model can be conceptualized as a labelled graph where different characteristics are used as follows:

1. The connection from state $X$ to state $Y$ carries some impactful weight $\omega_{x, y}$ called causal impact.

2. Number of incoming causal impacts $\omega_{x, y}$ $X(t)$ (usually from states $X$ to state $Y$ ) are combined using the combination function $\mathrm{c}_{Y}(.$.$) .$

3. Each state has a speed of change element (speed factor $\eta_{Y}$ ) to determine how fast the value of a particular state will change over time due to the received impact.

The above three characteristics are the main elements that define the temporal-causal relationships of a network model. A brief explanation of the network conceptual and numerical representations related to network terms is given below in Table 1.

The mentioned network characteristics in Table 1 are the core specifications that explain a temporal network modelling technique. These characteristics can serve as an input to a dedicated simulation environment after applying them to generate an appropriate function representing the dynamic behaviour between several states. For example, to compute the values of state $Y$ at different time points, the causal impacts imposed on $Y$ via incoming connections from other states at time $t$ and $t+\Delta t$ compute $Y$ values as follows.

$$
\begin{aligned}
Y(t+\Delta t) & =Y(t)+\eta_{Y .}\left[\operatorname{aggimpact}_{Y}(t)-Y(t)\right] .
\end{aligned}
$$


Table 2. Nomenclatures of related concepts

\begin{tabular}{|c|c|c|}
\hline No. & Concepts & Formalization \\
\hline 1 & Stressful event & $\mathrm{Se}$ \\
\hline 2 & Situational demands & $S d$ \\
\hline 3 & Resources & $R s$ \\
\hline 4 & Personality & $\mathrm{Pr}$ \\
\hline 5 & Social contact & $S c$ \\
\hline 6 & Self-efficacy & $S f$ \\
\hline 7 & Burden & $\mathrm{Br}$ \\
\hline 8 & Resilience & $R i$ \\
\hline 9 & Motivation & $M v$ \\
\hline 10 & Problem-focused & $P f$ \\
\hline 11 & Emotional-focused & $E f$ \\
\hline 12 & Cynicism & $C y$ \\
\hline 13 & Short-term stress & Sts \\
\hline 14 & Long-term stress & Lts \\
\hline 15 & $\begin{array}{c}\text { Short-term emotional } \\
\text { exhaustion }\end{array}$ & Ste \\
\hline 16 & $\begin{array}{l}\text { Long-term emotional } \\
\text { exhaustion }\end{array}$ & Lte \\
\hline 17 & Short-term burnout & $S t b$ \\
\hline 18 & Long-term burnout & $L t b$ \\
\hline
\end{tabular}

The above differential equation can be rewritten as:

$$
\left.\mathrm{d} Y(t) / \mathrm{dt}=\eta_{Y} . \operatorname{aggimpact}_{Y}(t)-Y(t)\right]
$$

Note here that the aggregation impact (aggimpactY $(t))$ is calculated as mentioned in Table 1.

$$
\begin{array}{r}
\operatorname{aggimpact}_{Y}(t)=\mathrm{c}_{Y}\left(\operatorname{impact}_{X l, Y}(t), \ldots,\right. \text { impact } \\
X k, Y(t))=\mathrm{c}_{Y}\left(\omega_{x l, y} X 1(t), \ldots, \omega_{x k, y} X k(t)\right)(3)
\end{array}
$$

\subsection{Conceptual model}

The first step towards developing the cognitive agent model is achieved by designing its conceptual representation in a causal graph based on the network-oriented modelling approach. As the concepts of burnout discussed in section 2, these concepts of burnout from research in psychological and cognitive sciences are conceptualised based on their causal interplays to form a complete visual understanding of the main elements that possibly cause different levels of burnout (low vs high) for the frontline professionals. The graphical overview of the conceptual model is displayed in Fig. 2.

Fig. 2 shows different components of the model. These components are grouped into four categories, namely 1) external variables, 2) burden evaluations, 3) appraisal and 4) burnout. Furthermore, the figure shows two types of nodes: the white nodes represent different model states while dark nodes represent the accumulative effects of the model's conditions.

\subsection{Formalization}

This section discusses utilizing the networkoriented modelling approach based on the temporal causal network to formalise the graphical representation of the burnout model shown in Fig. 3. In the formalisation, all nodes are linked to have a range of values between 0 (low) and 1 (high). Based on the interaction between these nodes, the new value of a state is determined throughout time. All the identified factors in section 2 are formalized as presented in Table 2.

The complete descriptions of the differential equations that construct the computational model of burnout is presented in the next subsections.

\subsubsection{Formalization of burden, resilience, and} motivation

In this formal model, burden impact $(B r)$ is computed based on the interplay between the available resources $(R s)$ and the level of resilience $(R i)$ to combat the imposed stressors of the event $(\mathrm{Se})$ and the accumulative effect of long-term emotional exhaustion (Lte). The level of burden can be seen as very intense when $\operatorname{Br}(\mathrm{t})=1$, and no impact of burden when $\operatorname{Br}(t)=0$. Resilience $(R i)$ is modelled based on the positive instantaneous interaction between personality $\operatorname{Pr}(t)$ and self-efficacy $(S f)$. The motivation level $(M v)$ is derived from the positive correlation between social contact $(S c)$ and personality $(P r)$.

$$
\begin{aligned}
& \operatorname{Br}(t)=\left[\mu_{b r} \cdot \operatorname{Se}(t)+\left(1-\mu_{b r}\right) \cdot \operatorname{Lte}(t)\right] \cdot\left[1-\left(\lambda_{r i}\right.\right. \\
& \left.\left.\operatorname{Ri}(t)+\left(1-\lambda_{r i}\right) \cdot \operatorname{RS}(t)\right)\right] \\
& \operatorname{Ri}(t)=\beta_{r i} S f(t)+\left(1-\beta_{r i}\right) \cdot \operatorname{Pr}(t) \\
& \operatorname{Mv}(t)=\alpha_{m v} \operatorname{Sc}(t)+\left(1-\alpha_{m v}\right) \cdot \operatorname{Pr}(t)
\end{aligned}
$$

Moreover, the parameters $\mu_{b r}, \lambda_{r i}, \beta_{r i}$, and $\alpha_{m v}$ represent the proportional change rate for all corresponding equations.

\subsubsection{Formalization of cynicism, problem focused, emotional focused}

Another condition is cynicism $(C y)$, which explains distant attitudes towards work. It is measured by the influence of burden $(B r)$, motivation $(\mathrm{Mv})$, self-efficacy $(\mathrm{Se})$, and the accumulative level of long-term emotional exhaustion (Lte). Both 


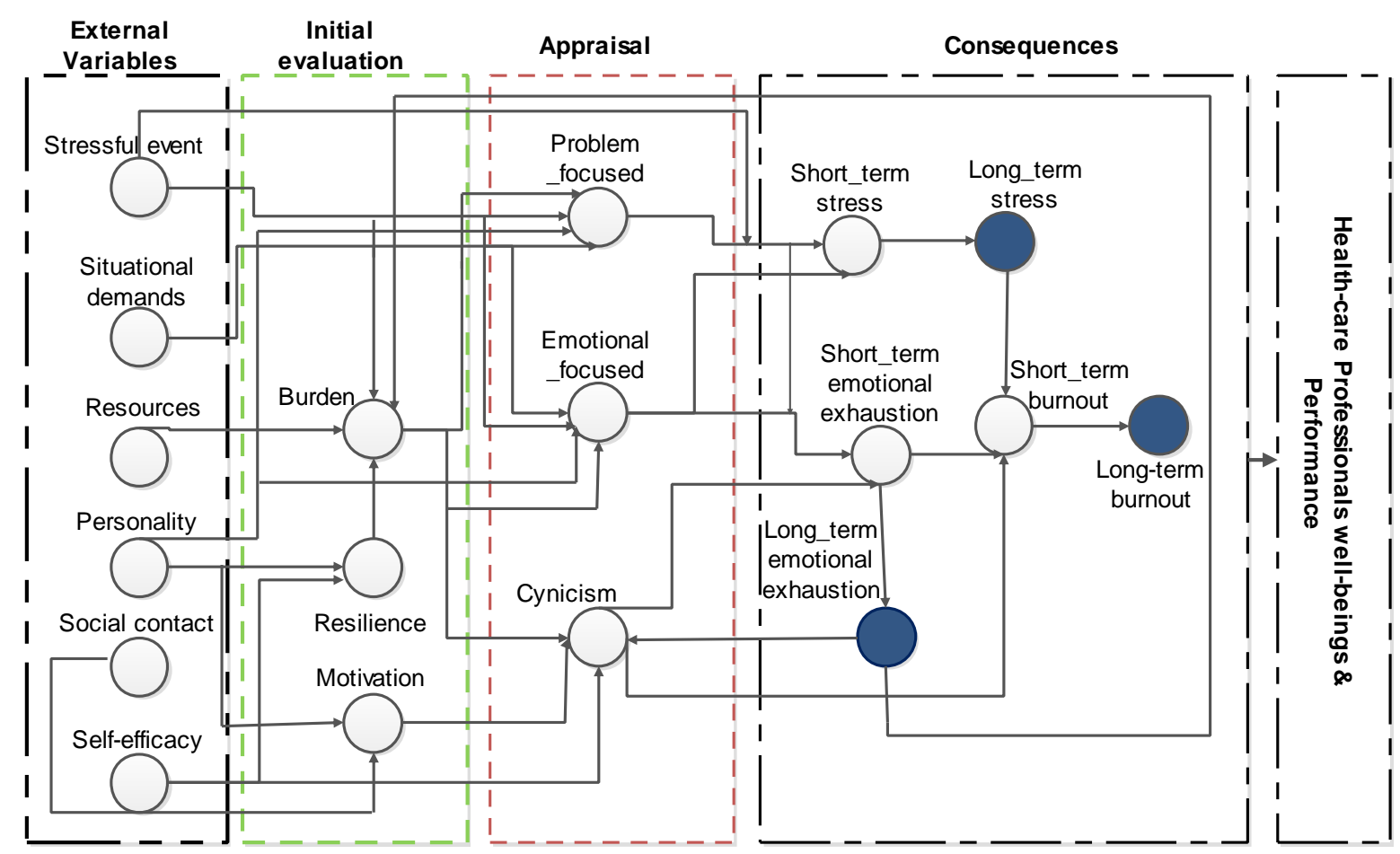

Figure. 2 Graphical representation model of cognitive agent model of burnout

motivation and self-efficacy levels contribute positively towards one's feeling of cynicism. In contrast, the burden and the accumulative level of long-term emotional exhaustion negatively impact cynicism.

$$
\begin{aligned}
C y(t)=\left[\left(\gamma_{c y} . \operatorname{Br}(t)+\left(1-\gamma_{c y}\right) . \text { Lte }(t)\right)\right] .\left[1-\left(\eta_{c y} .\right.\right. & \\
& \left.\left.\operatorname{Mv}(t)+\left(1-\eta_{c y}\right) . S f(t)\right)\right] \\
E f(t)= & {\left[\left(\mathrm{w}_{e f 1} . \operatorname{Br}(t)+\mathrm{w}_{e f 2 .} \operatorname{Se}(t)+\mathrm{w}_{e f 3 .} \operatorname{Sd}(t)\right)\right] .(1-} \\
& \operatorname{Pr}(t)), \text { where } \sum_{i=1}^{3} w_{e f i}=1
\end{aligned}
$$

$$
\begin{aligned}
\operatorname{Pf}(t)= & {\left[1-\left(\mathrm{w}_{p f 1} . \operatorname{Br}(t)+\mathrm{w}_{p f 2} . S e(t)+\mathrm{w}_{3 p f .} S d(t)\right)\right] . } \\
& (\operatorname{Pr}(t)), \text { where } \sum_{i=1}^{3} w_{p f i}=1
\end{aligned}
$$

Other essential conditions are emotional-focused $(E f)$ and problem-focused $(P f)$ strategies that control the stressful situation either by interpreting it as a problem that can be controlled or a situation that needs to be avoided emotionally, which later has negative consequences as stress and emotional exhaustion. Emotional focused (Ef) is calculated by simulating the potential positive effects throughout time $t$ using the weighted sum of three different elements namely, burden $(B r)$, stressful event $(\mathrm{Se})$, and situational demands $(S d)$. Furthermore, personality level $(P r)$ plays a key factor in controlling the level of emotional-focused as it is correlated negatively. Similarly, problem-focused is computed by the weighted sum of burden $(B r)$, stressful event $(\mathrm{Se})$, and situational demands (Sd). However, personality level $(\mathrm{Pr})$ is positively controlling the level of problem-focused. In addition, the parameters $\gamma_{c y}$ and $\eta_{c y}$ are used to regulate the respective equations.

\subsubsection{Formalization of short-term stress, short-term emotional exhaustion, short-term burnout}

Short-term stress $(\mathrm{Sts})$ is modelled by calculating the proportional contributions of stressful events $(\mathrm{Se})$ and emotional-focused (Ef). These two conditions contribute positively towards the development of short-term stress. However, the control ability towards the situation (i.e., problem-focused) reduces the progress in short-term stress. A similar calculating process is implemented to measure shortterm emotional exhaustion (Ste). The proportional contribution of cynicism $(C y)$ and emotional-focused $(E f)$ determine the value of short-term emotional exhaustion. Like short-term stress, problem-focused $(P f)$ curbs the growth of short-term emotional exhaustion.

$$
\begin{gathered}
S t s(t)=\left[\omega_{s t s .} S e(t)+\left(1-\omega_{s t s}\right) \cdot E f(t)\right] \cdot[(1-f(t))] \\
S t e(t)=\left[\lambda_{\text {ste. }} C y(t)+\left(1-\lambda_{s t e}\right) . E f(t)\right] .(1-P f(t))
\end{gathered}
$$


Table 3. Initial settings of the simulation experiments

\begin{tabular}{|c|c|c|c|}
\hline Exogenous Factors & $\mathbf{\# 1}$ & $\mathbf{\# 2}$ & $\mathbf{\# 3}$ \\
\hline Stressful event $(\mathrm{Se})$ & 1 & 0.9 & 0.1 \\
\hline Situational demands $(S d)$ & 1 & 0.9 & 0.1 \\
\hline Resources $(R s)$ & 0.1 & 0.5 & 0.9 \\
\hline Personality $(P r)$ & 0.1 & 0.5 & 0.9 \\
\hline Social contact $(S c)$ & 0.1 & 0.5 & 0.9 \\
\hline Self-efficacy $(S f)$ & 0.1 & 0.5 & 0.9 \\
\hline & & & \\
\hline
\end{tabular}

$S t b(t)=\left[\mathrm{w}_{s t b 1} . \operatorname{Lts}(t)+\mathrm{w}_{s t b 2 .} . S t e(t)+\mathrm{w}_{s t b 3 .} C y(t)\right]$ where $\sum_{i=1}^{3} w_{\text {stbi }}=1$

In the case of short-term burnout $(S t b)$, it describes the positive weighted sum of long-term stress (Lts), short-term emotional exhaustion (Ste), and cynicism $(C y)$. The combination of these three factors determines the overall level of short-term burnout. The weighted sum parameters $\mathrm{w}_{s t b 1}, \mathrm{w}_{s t b 2}$, $\mathrm{W}_{s t b 3}$ and regulatory parameters $\omega_{s t s}, \lambda_{s t e}$ are used to balance the contribution preferences.

\subsubsection{Formalization of long-term stress, long-term emotional exhaustion, and long-term burnout}

The accumulative values of the temporal relationships are measured based on the accumulated presence of the instantaneous relationships. For example, long-term stress (Lts) is mainly represented as the accumulation of exposure towards short-term stress $(S t s)$ in a time interval between $t$ and $t+\Delta t$. It means the long-term stress builds or reduces over time. When the short-term stress $(S t s)$ is higher than the previous long-term stress $(S t s-L t s)$, then the long-term stress increases and vice versa. This explanation can also be applied to other circumstances that represent the temporal phenomenon, such as long-term emotional exhaustion (Lte) and long-term burnout (Ltb).

$$
\begin{aligned}
& L t s(t+\delta t)=L t s(t)+\eta_{l t s .}[S t s(t)-L t s(t)] .(1- \\
& \operatorname{Lts}(t)) . \operatorname{Lts}(t) . \delta t
\end{aligned}
$$

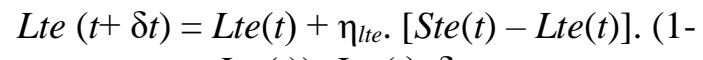

$$
\begin{aligned}
& \operatorname{Lte}(t)) . \operatorname{Lte}(t) . \delta t \\
& L t b(t+\delta t)=L t b(t)+\eta_{l t b .}[S t b(t)-L t b(t)] .(1- \\
& L t b(t)) . L t b(\mathrm{t}) . \delta t
\end{aligned}
$$

Note that the rates of change for the temporal factors are computed by the flexibility rates $\eta$ lts, $\eta$ lte, and $\eta$ ltb. Furthermore, some parts of the above equations were used to regulate the temporal boundary values. For example, the part $((1-L t s(t))$. $L t s(t))$ expresses the boundary limit of long-term stress (either $L t s=0$ or $L t s=1$ ). All the derived equations serve as an input to a numerical simulation environment to generate simulation traces for experimental purposes. These traces explain why healthcare professionals develop burnout while combating covid-19.

\section{Analysis by simulation experiments}

This section shows the results of the simulation experiments that illustrate the developmental process (increase vs decrease) of burnout for healthcare professionals while facing the impact of the covid-19 crisis. Moreover, a numerical simulation environment (i.e., matlab and ms excel) is used to attain the experiment. It is worth noting that all the exogenous factors of burnout are static in the simulation (ranging between 0 (low) and 1 (high)). However, all the other factors in the model are temporal, which changes over time due to the interactions among these factors. For example, the value of burden $(\mathrm{Br})$ of the previous time-step is taken into account of the next time point. Due to a large number of possible scenarios that can be simulated, only three scenarios are selected which show the changes in burnout values. These scenarios are; 1) High impact of burnout, 2) moderate impact of burnout and 3) low impact of burnout. Furthermore, the initial settings of the external factors for the three scenarios are presented in Table 3. In addition to the initial values of the external factors, all the change rate and regulatory parameters are also specified as the followings; time-steps $=500$ (expresses the duration of the event which approximately last for one week), $\alpha \mathrm{mv}=0.5, \mu_{b r}=0.7$, $\beta_{r i}=0.5, \gamma_{c y}=0.9, \eta_{c y}=0.2, \lambda_{s t e}=0.5, \eta_{l t s}=0.6, \eta_{l t e}=0.6$, $\delta \mathrm{t}=0.4$ and $\eta_{l t b}=0.6$.

\subsection{Scenario \#1: High impact of burnout}

For simulating the high impact of burnout, healthcare professionals who face the crisis encounter a seriously stressful event that demands them much to tackle the developed burden and psychological ramifications such as stress and emotional exhaustion. Moreover, they do not have positive personalities, enough social support, and adequate resources that help them control the adverse impact of the burdening event. These conditions make healthcare professionals apply emotionalfocused strategies to cope with the consequences rather than problem-focused, and this situation contributes to increasing the disengagement level (i.e., cynicism). 

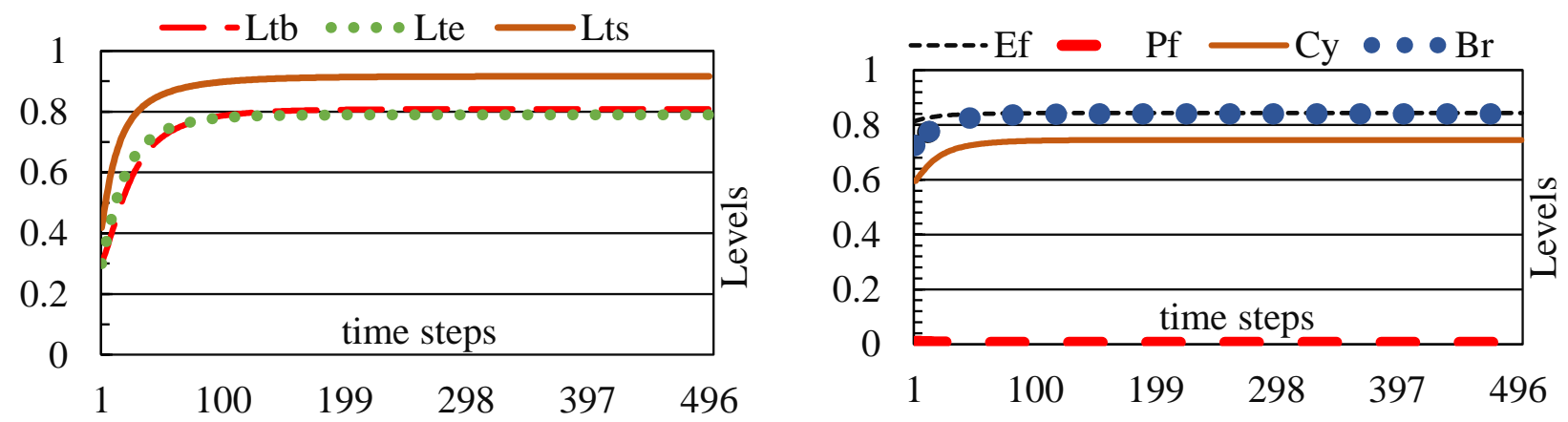

(a)
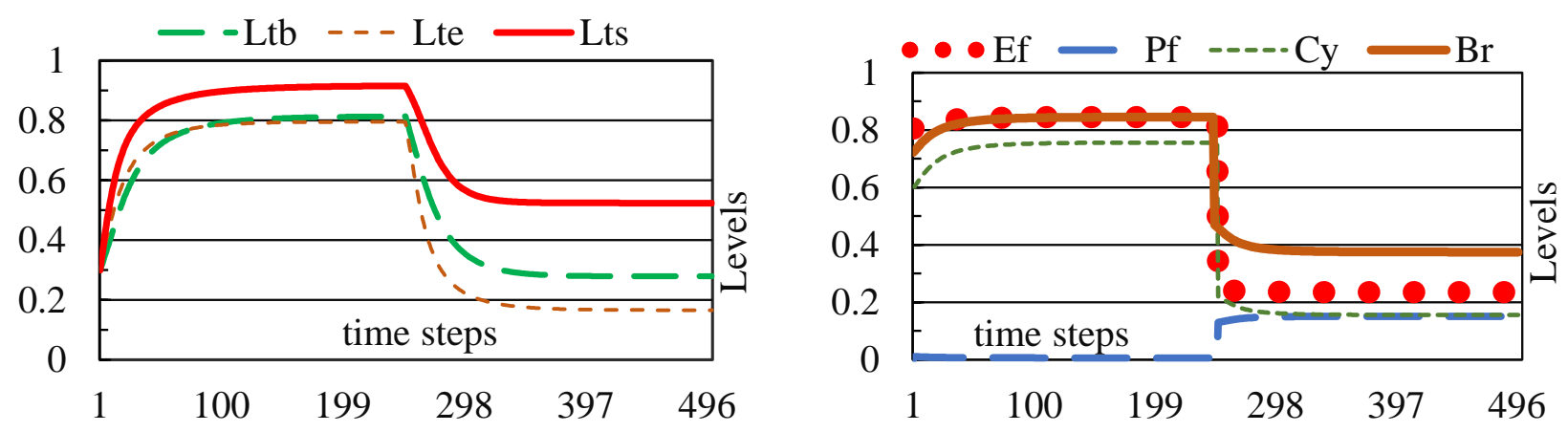

(b)
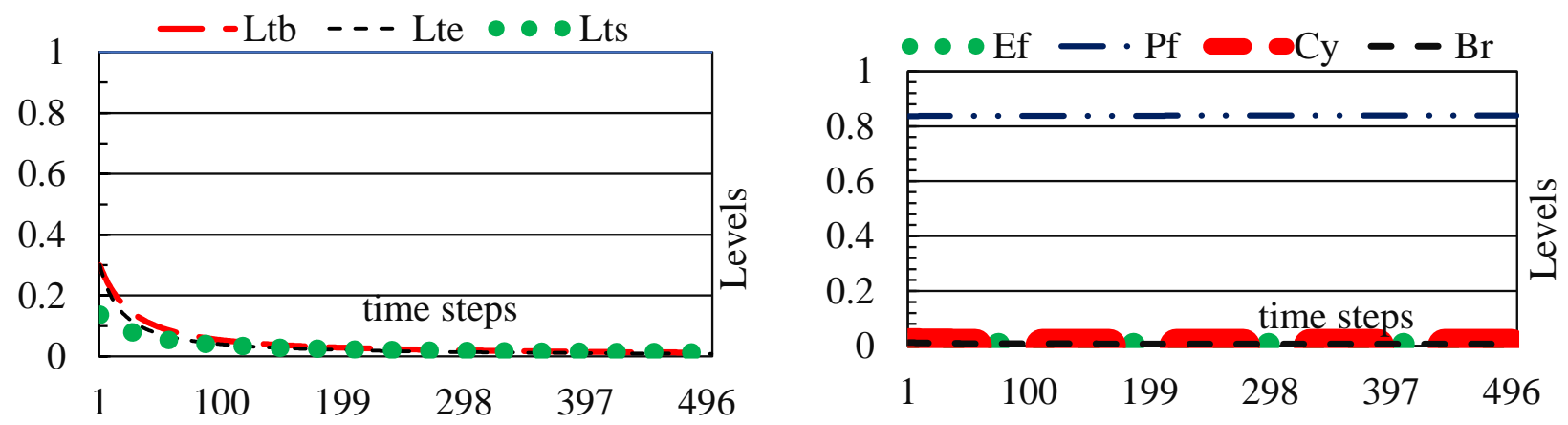

(c)

Figure. 3 Simulation results: (a) high impact of burnout, (b) moderate impact of burnout, and (c) low impact of burnout

\subsection{Scenario \#2: Moderate impact of burnout}

For a balanced effect of burnout, albeit healthcare professionals usually deal with a stressful crisis that demands them, they can regulate the adverse impact of burnout development when they have a moderate level of resources, social contact, and personality. Furthermore, they have a reasonable belief in their capacity to control the negative impacts (i.e., intermediate level of self-efficacy).

\subsection{Scenario \#3: Low impact of burnout}

Obviously, healthcare professionals who are having enough social support from their colleagues, friends, and family members tend to be highly motivated and this makes them have a high level of motivation that increases their cynicism level.
Moreover, in this scenario, they have positive beliefs, positive personalities, and enough resources to fight the demanding stressful event. These high levels make them positively face the crisis via adopting a problem-focused strategy that later curbs the adverse impacts of long-term stress, long-term emotional exhaustion, and long-term burnout. Fig. 5 shows the simulation experiment of low impact of burnout.

To wrap up the simulation results, all the simulation traces illustrated in Fig. 3 satisfactorily explain the identified causal relationships as summarised in section 2 .

\section{Mathematical analysis}

Dynamic agent models that develop based on the network-oriented modelling technique can be mathematically analysed to determine the stationary 
or equilibrium points [27-28]. Discovering the equilibria or the stationary points of the mathematical model considers as an excellent reasonable indicator for the theoretical correctness of the formal specifications. As a first step towards analysing the equilibria, those formal specifications are replaced with values such that the differences between time point $t$ and $t+\delta t$ are all 0 (in particular, all temporal relationships). One crucial assumption should be made; all exogenous variables have a constant value. Assuming all parameters are non-zero, this leads to the following equations where an equilibrium state is characterised by:

$$
\begin{aligned}
& M v=\alpha_{m v} . S c+\left(1-\alpha_{m v}\right) \cdot P r \\
& R i=\beta_{r i} . S f+\left(1-\beta_{r i}\right) . P r \\
& B r=\left[\mu_{b r} . S e+\left(1-\mu_{b r}\right) . L t e\right] .\left[1-\left(\lambda_{r i .} R i+\left(1-\lambda_{r i}\right)\right. \text {. }\right. \\
& R s) \text { ] } \\
& C y=\left[\left(\gamma_{c y} . B r+\left(1-\gamma_{c y}\right) \cdot L t e\right)\right] \cdot\left[1-\left(\eta_{c y} . M v\right.\right. \\
& \left.\left.+\left(1-\quad \eta_{c y}\right) \cdot S f\right)\right] \\
& E f=\left[\left(\mathrm{w}_{e f 1} . B r+\mathrm{w}_{e f 2} . S e+\mathrm{w}_{e f 3 .} . S d\right)\right] .(1-P r) \\
& P f=\left[1-\left(\mathrm{w}_{p f 1} . B r+\mathrm{w}_{p f 2} . S e+\mathrm{w}_{3 p f} . S d\right)\right] .(P r) \\
& S t s=\left[\omega_{s t s} \cdot S e+\left(1-\omega_{s t s}\right) \cdot E f\right] \cdot[(1-P f)] \\
& S t e=\left[\lambda_{\text {ste. }} . C y+\left(1-\lambda_{\text {ste }}\right) . E f\right] .(1-P f) \\
& S t b=\left[\mathrm{w}_{s t b 1} . L t s+\mathrm{w}_{s t b 2 .} . S t e+\mathrm{w}_{s t b 3 .} . C y\right] \\
& \eta_{l t s .}[S t s-L t s] .(1-L t s) . L t s=0 \\
& \eta_{l t e .}[S t e-L t e] .(1-L t e) . L t e=0 \\
& \eta_{l t b .}[S t b-L t b] .(1-L t b) . L t b=0
\end{aligned}
$$

Assuming adaptation rates are equal to 1 . From the Eqs. (25) to (27), the following cases are identified,

$$
\begin{gathered}
(S t s=L t s) \vee(L t s=1) \vee(L t s=0)(S t e=L t e) \vee(L t e=1) \\
\vee(L t e=0)(S t b=L t b) \vee(L t b=1) \vee(L t b=0) \quad(28)
\end{gathered}
$$

Hence, a first conclusion can be obtained where the stability points can only occur when Sts = Lts, or $L t s=1$, or $L t s=0$ (as in Eq. (25)). Thus, if these three conditions were combined, then a new set of relationships as in $(\mathrm{A} \vee \mathrm{B} \vee \mathrm{C}) \wedge(\mathrm{D} \vee \mathrm{E} \vee \mathrm{F})$ expression can be formed:

$$
\begin{aligned}
& (S t s=L t s \square L t s=1 \vee L t s=0) \wedge(\text { Ste }=L t e \vee L t e=1 \\
& \vee L t e=0) \wedge(S t b=L t b \vee L t b=1 \vee t b=0)
\end{aligned}
$$

This expression can be elaborated using law of distributivity as $(\mathrm{A} \wedge \mathrm{D}) \vee(\mathrm{A} \wedge \mathrm{E}) \vee(\mathrm{A} \wedge \mathrm{F}) \vee, \ldots, \vee$ $(\mathrm{C} \wedge \mathrm{F})$ and this will result:

$$
\begin{aligned}
(S t s & =L t s \wedge \text { Ste }=L t e \wedge S t b=L t b) \vee, \ldots,(\text { Lts }=0 \\
\wedge L t e & =0 \wedge L t b=0)
\end{aligned}
$$

Eq. (30) provides twenty-seven possible combinations of equilibria points (in this case, $3^{3}$ possibilities) to be further analysed. Due to many possible combinations, only three equilibria cases will be explored.

\section{Case \#1: Lts $=$ Sts $\wedge$ Lte $=1 \wedge L t b=1$}

For this case, from equations (Eq. (4) to Eq. (12)), Eq.4 results,

$$
B r=\left[\mu_{b r} . S e+\left(1-\mu_{b r}\right)\right] .\left[1-\left(\lambda_{r i} R i+\left(1-\lambda_{r i}\right) . R s\right)\right]
$$

Assuming $\mu_{b r}=0.5$ and $\lambda_{r i}=0.5$ (i.e., to represent an equal contribution for both sides), leads to,

$$
B r=[S e] .[1-(R i+R s)]
$$

Moreover, by Eq. (7), it follows.

$$
\left.\left.C y=\underset{S f)]}{\left[\left(\gamma_{c y} .\right.\right.} B r+\left(1-\gamma_{c y}\right)\right)\right] .\left[1-\left(\eta_{c y} . M v+\left(1-\eta_{c y}\right) .\right.\right.
$$

Assuming $\gamma_{c y}=0.5$ and $\eta_{c y}=0.5$, this leads to,

$$
C y=(B r) \cdot[1-(M v+S f)]
$$

From Eq. (12), it follows.

$$
S t b=\left[\mathrm{w}_{s t b l}+\mathrm{w}_{s t b 2 .} . S t e+\mathrm{w}_{s t b 3 .} C y\right]
$$

Assuming the sum of the regulatory parameters is equal to1, it results,

$$
S t b=S t e+C y
$$

Note that after simulating the analysis result, the simulation experiment showed that the model works correctly as the stability points were seen as depicted in Fig 4. The same principle has been implemented in the following case.

\section{Case \#2: Lte $=$ Ste $\wedge$ Lts $=1 \wedge L t b=0$}

From Eq. (4), it follows that this is equivalent to:

$$
\begin{aligned}
& B r=\left[\mu_{b r} . S e+\left(1-\mu_{b r}\right) . S t e\right] .\left[1-\left(\lambda_{r i .} R i+\left(1-\lambda_{r i}\right) .\right.\right. \\
& R s)]
\end{aligned}
$$



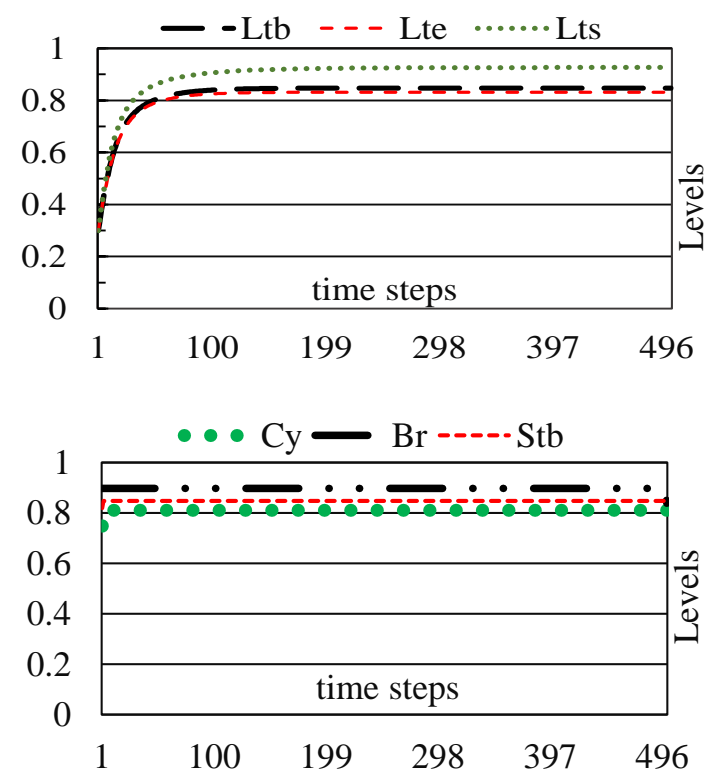

Figure. 4 Visual representation of case\#1

Assuming $\mu_{b r}=0.5$ and $\lambda_{r i}=0.5$, it results,

$$
B r=[S e+S t e] .[1-(R i+R s)]
$$

And hence by Eq. (7),

$$
C y=[(B r+S t e)] .[1-(M v+S f)]
$$

Moreover, from Eq. (12), it follows,

$$
S t b=S t e+C y
$$

\section{Automated analysis}

This section illustrates the internal validity process of the constructed cognitive agent model of burnout. Since no numerical data can be easily obtained to explain the dynamic causal behaviours of the cognitive agent, certain behaviours or patterns can still be found in the literature based on empirical studies that verify against simulated behaviours to prove the internal validity of the developed model [29]. In other words, the model can be verified to ensure its results are indeed adhering to psychological and cognitive literature. To attain this, a temporal trace language (TTL) has been developed to check whether the model behaves as it should be or not by running several simulations and verifying them against the patterns available in the literature and previous empirical studies [30]. The importance of TTL resides in its supports for the specification of both qualitative and quantitative aspects and subsumes specification languages based on differential equations. It allows explicit reference to time points and time durations, which enables the modelling of the dynamics of continuous real-time phenomena [see ref for extensive details]. This dedicated language is built on atoms state $(\gamma, t) \mid=p$, meaning that state property $\mathrm{P}$ holds in trace $\gamma$ (simulation points of states over time). Using this concept, many simulation traces including the one shown in section 4 are used to be verified against the identified patterns from the literature, and the results were confirmed.

P1- Burnout level is high due to a high level of stress [31].

For all time points $t 1$ and $t 2$ in trace $\gamma$,

If and at time point $t 2$ the level of $L t s$ is s2, and at time point $t 1$ the level of $L t b$ is b1, and at time point $\mathrm{t} 2$ the level of $L t b$ is $\mathrm{b} 2$, and $\mathrm{s} 2>=\mathrm{s} 1$, and $\mathrm{t} 1<=\mathrm{t} 2$, then $\quad b 2>=b 1$

$\forall \gamma$ : TRACE, $\forall t 1, t 2$ : TIME, $\forall s 1, s 2, b 1, b 2$ : REAL state $(\gamma, t 1) \mid=$ has_value (long_term_stress, $s 1) \&$ state $(\gamma, t 2) \mid=$ has_value (long_term_stress, s2) \& state $(\gamma, t 1) \mid=$ has_value (long_term_burnout, $b 1) \&$ state $(\gamma, t 2) \mid=$ has_value (long_term_burnout, $b 2) \&$ s $2 \geq$ $s 1 \& b 2 \geq b 1 \& t 2 \geq t 1 \Rightarrow b 2 \geq b 1$

Property $\mathrm{p} 1$ can be used to check whether the high level of stress contributes to the development of burnout. The property succeeded on the traces (i.e., simulation results from matlab) so that the burnout level was increasing due to stress increment.

P2- Healthcare workers with a high level of psychological resilience tend to control the increment of burnout symptoms in their risky working environment [23].

For all time points $t 1$ and $t 2$ in trace $\gamma$,

If at time point $t 1$ the level of Ri is $r 1$,

and at time point $t 2$ the level of $\mathrm{Ri}$ is $r 2$,

and at time point $t 1$ the level of Ltb is $b 1$,

and at time point $t 2$ the level of Ltb is $b 2$,

and $\quad r 2 \geq r 1$, and $t 1 \leq t 2$,

then $\quad b 2 \leq b 1$

$\forall \gamma$ : TRACE, $\forall t 1, t 2$ : TIME, $\forall r 1, r 2, b 1, b 2$ : REAL $\operatorname{state}(\gamma, t 1) \mid=$ has_value (resilience, $r 1) \&$ $\operatorname{state}(\gamma, t 2) \mid=$ has_value (resilience, $r 2) \&$ $\operatorname{state}(\gamma, t 1) \mid=$ has_value (long_term_burnout, $b 1) \&$ $\operatorname{state}(\gamma, t 2) \mid=$ has_value (long_term_burnout, b2) \& $s 2 \geq s 1 \& b 1 \leq b 2 \& t 1 \leq t 2 \Rightarrow b 2 \leq b 1$

Property P2 discusses how psychological resilience can impact the development of burnout. It shows that high level of resilience can reduce the 
impact of burnout and it succeeded on the simulation traces. This identified property from the literature also makes sure the model is correct.

P3- Cynicism or disengagement of the work leads to a high level of burnout [32].

For all time points $t 1$ and $t 2$ in trace $\gamma$,

If at time point $t 1$ the level of $C y$ is $c$,

and at time point $\mathrm{t} 2$ the level of $L t b$ is $b$,

and $\quad c 1 \geq 0.5$ and $t 2>=18$,

then $\quad b 2>=0.5$

$\forall \gamma$ : TRACE, $\forall \mathrm{t} 1, \mathrm{t} 2$ : TIME, $\forall \mathrm{c}, \mathrm{b}$ : REAL

state $(\gamma, t 1) \mid=$ has_value (cynicism, $c) \&$

state $(\gamma, t 2) \mid=$ has_value (long_term_burnout, $b) \&$

$c \geq 0.5 \& t 2 \geq 18 \Rightarrow b \geq 0.5$

This property P3 can be used to check whether the high level of cynicism (equal or greater than 0.5 ) can lead to a high level of burnout $(>=0.5)$. The property succeeded on the traces as burnout increased to above 0.5 and cynicism level was above 0.5 after eighteentime points.

\section{Conclusions}

This paper contributes in developing a computational cognitive agent model of burnout for front-line healthcare professionals using the networkoriented modelling approach based on temporal causal networks. A number of burnout determinants and their causal interplays were identified from the literature and linked to construct the model. In addition, differential equations were implemented to formalise the model and generate an executable model for simulation purposes. Hence, several simulation experiments were conducted to understand the model's behaviours. Simulation results proved that the model behaves as expected and in line with the literature. Moreover, by mathematical analysis, the equilibria of the model have been specified, and a number of selected cases were checked to prove the correct implementation of the model. In addition, automated logical verification using temporal trace language (TTL) was used to verify the model's validity. Thus, using generated simulation traces, the model has been verified against a number of properties describing emerging patterns put forward in the literature. In addition, the model could be used as the basis to develop intelligent supportive agents that are capable of helping frontline workers in combatting burnout effect. For future work, efforts are required to solidify the model by exploring more related factors and performing parameter tuning to make the model fits a different range of individuals.

\section{Conflicts of interest}

The authors declare no conflict of interest.

\section{Author contributions}

The first author has done background work, conceptualization, methodology, implementation, result analysis, preparing and editing the draft, and visualization. The second author has done the supervision, review of work and project administration.

\section{References}

[1] E. Stępień, M. Koleśnik, K. Mitura, M. Malm, B. Drop, M. Jędrych, and M. Polz-Dacewicz, "SARS-CoV-2 Infection Prevalence in the Population of South-Eastern Poland", Diagnostics, Vol. 11, No. 11, p. 2115, 2021.

[2] M. D. Braquehais, S. V. Cáceres, E. G. Durán, G. Nieva, M. Casas, and E. Bruguera, "The impact of the COVID-19 pandemic on the mental health of healthcare professionals", $Q J M$ An Int. J. Med., Vol. 113, No. 9, pp. 613-617, Sep. 2020, doi: 10.1093/qjmed/hcaa207.

[3] G. Zerbini, A. Ebigbo, P. Reicherts, M. Kunz, and H. Messman, "Psychosocial burden of healthcare professionals in times of COVID-19 - a survey conducted at the University Hospital Augsburg", Ger. Med. Sci., Vol. 18, pp. Doc05Doc05, 2020, doi: 10.3205/000281.

[4] F. Dong, H. Liu, M. Yang, C. Lu, N. Dai, Y. Zhang, N. Robinson, and J. Liu., "Immediate Psychosocial Impact on Healthcare Workers During COVID-19 Pandemic in China: A Systematic Review and Meta-Analysis", Frontiers in Psychology, Vol. 12. p. 1503, 2021, [Online].

Available: https://www.frontiersin.org/article/10.3389/fpsy g.2021.645460.

[5] H. M. Alrawashdeh, "Occupational burnout and job satisfaction among physicians in times of COVID-19 crisis: a convergent parallel mixedmethod study", BMC Public Health, Vol. 21, No. 1, p. 811, 2021, doi: 10.1186/s12889-02110897-4.

[6] L. P. Morera, J. I. Gallea, M. A. Trógolo, M. E. Guido, and L. A. Medrano, "From Work WellBeing to Burnout: A Hypothetical Phase Model", Front. Neurosci., Vol. 14, p. 360, 2020, doi: 10.3389/fnins.2020.00360.

[7] C. Maslach and S. E. Jackson, "The 
measurement of experienced burnout", J. Organ. Behav., Vol. 2, No. 2, pp. 99-113, 1981, doi: https://doi.org/10.1002/job.4030020205.

[8] Z. Dujmić, E. Machielse, and J. Treur, "A Temporal-Causal Modeling Approach to the Dynamics of a Burnout and the Role of Physical Exercise", In: Proc. of the Ninth Annual Meeting of the BICA Society, Prague, Czech Republic, pp. 88-100, 2018.

[9] M. Maijer, E. Solak, and J. Treur, "An adaptive network model for burnout and dreaming", In: Proc. of the 20th International Conference on Computational Science, ICCS'20, Amsterdam, the Netherlands, Vol. 12137, pp. 342-356, 2020.

[10] F. Squazzoni, J. Polhill, B. Edmonds, P. Ahrweiler, P. Antosz, G. Scholz, M. Borit, H. Verhagen, F. Giardini, and N. Gilbert, "Computational Models That Matter During a Global Pandemic Outbreak: A Call to Action", $J$. Artif. Soc. Soc. Simul., Vol. 23, No. 2, p. 10, 2020, doi: 10.18564/jasss.4298.

[11] J. Treur, "Network-Oriented Modeling and Its Conceptual Foundations BT - Network-Oriented Modeling: Addressing Complexity of Cognitive, Affective and Social Interactions", J. Treur, Ed. Cham: Springer International Publishing, 2016.

[12] A. A. Aziz and H. M. A. Ghanimi, "Reading with Robots: A Personalized Robot-Based Learning Companion for Solving Cognitively Demanding Tasks", Int. J. Adv. Sci. Eng. Inf. Technol., Vol. 10, pp. 1489-1496, 2020.

[13] T. Bosse, M. Hoogendoorn, M. Klein, R. Van Lambalgen, P. P. V. Maanen, and J. Treur, "Incorporating human aspects in ambient intelligence and smart environments", Handbook of Research on Ambient Intelligence and Smart Environments: Trends and Perspectives, IGI Global, pp. 128-164, 2011.

[14] T. Bosse, F. Both, C. Gerritsen, M. Hoogendoorn, and J. Treur, "Methods for Model-Based Reasoning within Agent-Based Ambient Intelligence Applications", Know.Based Syst., Vol. 27, pp. 190-210, 2012, doi: 10.1016/j.knosys.2011.09.011.

[15] R. Duell, M. Hoogendoorn, M. Klein, and J. Treur, "An Ambient Intelligent Agent Model Using Controlled Model-Based Reasoning to Determine Causes and Remedies for Monitored Problems", In: Proc. of 2008 IEEE/WIC/ACM International Conference on Web Intelligence and Intelligent Agent Technology, Vol. 3, pp. 489-494, 2008, doi: 10.1109/WIIAT.2008.322.

[16] J. E. M. Jiménez, L. M. B. Donoso, M. C. Fernández, S. B. Hofheinz, B. M. Jiménez, and E. Garrosa, "The Job Demands and Resources
Related to COVID-19 in Predicting Emotional Exhaustion and Secondary Traumatic Stress Among Health Professionals in Spain", Frontiers in Psychology, Vol. 12. p. 612, 2021, [Online]. Available: https://www.frontiersin.org/article/10.3389/fpsy g.2021.564036.

[17] A. Koontalay, W. Suksatan, K. Prabsangob, and J. M. Sadang, "Healthcare Workers' Burdens During the COVID-19 Pandemic: A Qualitative Systematic Review", J. Multidiscip. Healthc., Vol. 14, pp. 3015-3025, 2021, doi: 10.2147/JMDH.S330041.

[18] H. H. Lee and J. A. Cranford, "Does resilience moderate the associations between parental problem drinking and adolescents' internalizing and externalizing behaviors? A study of Korean adolescents", Drug Alcohol Depend., Vol. 96, No. 3, pp. 213-221, 2008, doi: 10.1016/j.drugalcdep.2008.03.007.

[19] N. Rivas, M. López, M. Castro, S. L. Vian, M. F. Castro, M. Cao,S. García, and J. Jiménez, "Analysis of Burnout Syndrome and Resilience in Nurses throughout the COVID-19 Pandemic: A Cross-Sectional Study", International Journal of Environmental Research and Public Health, Vol. 18, No. 19. 2021, doi: 10.3390/ijerph181910470.

[20] W. Zeng, X. Wu, Y. Xu, J. Wu, Y. Zeng, J. Shao, D. Huang, and Z. Zhu, "The Impact of General Self-Efficacy on Psychological Resilience During the COVID-19 Pandemic: The Mediating Role of Posttraumatic Growth and the Moderating Role of Deliberate Rumination", Frontiers in Psychology, Vol. 12. p. 2326, 2021, [Online].

Available: https://www.frontiersin.org/article/10.3389/fpsy g.2021.684354.

[21] A. Oshio, K. Taku, M. Hirano, and G. Saeed, "Resilience and Big Five personality traits: A meta-analysis", Pers. Individ. Dif., Vol. 127, pp. 54-60, 2018, doi: https://doi.org/10.1016/j.paid.2018.01.048.

[22] H. Blake, F. Bermingham, G. Johnson, and A. Tabner, "Mitigating the Psychological Impact of COVID-19 on Healthcare Workers: A Digital Learning Package", International Journal of Environmental Research and Public Health, Vol. 17, No. 9. 2020, doi: 10.3390/ijerph17092997.

[23] C. D. Monte, S. Monaco, R. Mariani, and M. D. Trani, "From Resilience to Burnout: Psychological Features of Italian General Practitioners During COVID-19 Emergency", Front. Psychol., Vol. 11, p. 567201, 2020, doi: 
10.3389/fpsyg.2020.567201.

[24] S. Aljhani, H. Alharbi, S. Aljameli, L. Hameed, K. Alaql, and M. Alsulaimi, "Burnout and coping among healthcare providers working in Saudi Arabia during the COVID-19 pandemic", Middle East Curr. Psychiatry, Ain Shams Univ., Vol. 28, No. 1, p. 29, 2021, doi: 10.1186/s43045-021-00108-6.

[25] Y. Zhang, C. Wang, W. Pan, J. Zheng, J. Gao, X. Huang, S. Cai, Y. Zhai, J. Latour, and C. Zhu, "Stress, Burnout, and Coping Strategies of Frontline Nurses During the COVID-19 Epidemic in Wuhan and Shanghai, China", Front. psychiatry, Vol. 11, p. 565520, 2020, doi: 10.3389/fpsyt.2020.565520.

[26] F. Jabeen, C. Gerritsen, and J. Treur, "Healing the next generation: an adaptive agent model for the effects of parental narcissism", Brain Informatics, Vol. 8, No. 1, p. 4, 2021, doi: 10.1186/s40708-020-00115-z.

[27] H. M. A. Ghanimi, A. H. Shnain, W. H. Ali, and L. L. S. Yi, "Computational Analysis and Evaluation for An Agent-Based Model of Stress Among Flood Victims", J. Crit. Rev., Vol. 7, No. 8, pp. 1320-1323, 2020.

[28] H. M. A. Ghanimi and A. A. Aziz, "Computational Analysis of Dynamics in an Agent-Based Model of Cognitive Load and Reading Performance", In: Proc. of International Conference of Reliable Information and Communication Technology, Kuala Lumpur, Malaysia, pp. 207-220, 2018.

[29] J. Treur, "What Is Happening BT - NetworkOriented Modeling: Addressing Complexity of Cognitive, Affective and Social Interactions", $J$. Treur, Ed. Cham: Springer International Publishing, pp. 349-391, 2016.

[30] T. Bosse, C. M. Jonker, L. V. D. Meij, A. Sharpanskykh, and J. Treur, "Specification and verification of dynamics in agent models", Int. J. Coop. Inf. Syst., Vol. 18, No. 1, pp. 167-193, 2009.

[31] M. Yıldırım and F. Solmaz, "COVID-19 burnout, COVID-19 stress and resilience: Initial psychometric properties of COVID-19 Burnout Scale", Death Studies, pp. 1-9, 2020, 10.1080/07481187.2020.1818885.

[32] D. Li, Y. Wang, H. Yu, Z. Duan, K. Peng, N. Wang, Q. Zhou, X. Hu, K. Fang, A. Wilson, J. $\mathrm{Ou}$, and $\mathrm{X}$. Wang, "Occupational Burnout Among Frontline Health Professionals in a High-Risk Area During the COVID-19 Outbreak: A Structural Equation Model", Front. Psychiatry, Vol. 12, p. 575005, 2021, doi: 10.3389/fpsyt.2021.575005. 